\title{
REVIEWS
}

\section{Empirical Consequences of Current Recommendations for the Design and Interpretation of Noninferiority Trials}

\author{
Scott K. Aberegg, MD, MPH', Andrew M. Hersh, MD' , and Matthew H. Samore, MD ${ }^{2}$
}

'Pulmonary Division, University of Utah School of Medicine, Salt Lake City, UT, USA; '2Division of Epidemiology, University of Utah School of Medicine, Salt Lake City, UT, USA.

BACKGROUND: Noninferiority trials are increasingly common, though they have less standardized designs and their interpretation is less familiar to clinicians than superiority trials.

OBJECTIVE: To empirically evaluate a cohort of noninferiority trials to determine 1) their interpretation as recommended by CONSORT, 2) choice of alpha threshold and its sidedness, and 3) differences between methods of analysis such as intention-to-treat and per-protocol.

DESIGN: We searched MEDLINE for parallel-group randomized controlled noninferiority trials published in the five highest-impact general medical journals between 2011 and 2016.

MAIN MEASURES: Data abstracted included trial design parameters, results, and interpretation of results based on CONSORT recommendations.

KEY RESULTS: One hundred sixty-three trials and 182 noninferiority comparisons were included in our analysis. Based on CONSORT-recommended interpretation, $79 \%$ of experimental therapies met criteria for noninferiority, 13\% met criteria for superiority, 20\% were declared inconclusive, and $2 \%$ met criteria for inferiority. However, for $12 \%$ of trials, the experimental therapy was statistically significantly worse than the active control, but CONSORT recommended an interpretation of inconclusive or noninferior. A two-sided alpha equivalent of greater than 0.05 was used in $34 \%$ of the trials, and in five of these trials, the use of a standard two-sided alpha of 0.05 led to changes in the interpretation of results that disfavored the experimental therapy. In four of the five comparisons where different methods of analysis (e.g., intention-to-treat and per-protocol) yielded different results, the intention-to-treat analysis was the more conservative. In $11 \%$ of trials, a secondary advantage of the new therapy was neither reported nor could it be inferred by reviewers.

CONCLUSIONS: In this cohort, the design and interpretation of noninferiority trials led to significant and systematic bias in favor of the experimental therapy. Clinicians should exercise caution when interpreting these trials. Future trials may be more reliable if design parameters are standardized.

J Gen Intern Med 33(1):88-96

DOI: $10.1007 / \mathrm{s} 11606-017-4161-4$

(c) The Author(s) 2017. This article is an open access publication

Received July 19, 2017

Revised July 28, 2017

Accepted August 9, 2017

Published online September 5, 2017

\section{INTRODUCTION}

Noninferiority trials are used to compare a new therapy (NT) to an active control (AC) when the use of a placebo control is not ethically feasible. The prevalence of noninferiority trials is increasing, 1,2 despite concerns about their validity owing to several design and interpretation controversies. ${ }^{3,}{ }^{4}$ Compared to superiority trials, the interpretation of noninferiority trials is less straightforward, and there is greater flexibility and variability in their design parameters. Little empirical research has evaluated how these unique features may affect the validity of noninferiority trial results and conclusions.

In 2006, the CONSORT [Consolidated Standards of Reporting Trials] Group provided specific recommendations for the reporting of noninferiority and equivalence trials. ${ }^{5,6}$ Our Figure 1 is a simulacrum of the CONSORT schematic. As explained in the caption, asymmetry in interpretation using this schematic creates a potential bias in favor of the new or experimental therapy. ${ }^{7}$ The frequency with which biased interpretation results from this asymmetry is unknown.

In a superiority trial, a two-sided alpha threshold for statistical significance is conventionally set at $<0.05$, whereas alpha thresholds in noninferiority trials are more variable. ${ }^{6}$ No investigations to date have evaluated whether the selection of an alpha threshold less stringent than a two-sided 0.05 equivalent influences the conclusions of noninferiority trials.

It is commonly noted that intention-to-treat analyses, recommended for superiority trials, are less conservative than perprotocol or as-treated analyses in noninferiority trials. ${ }^{6,}{ }^{8-11}$ However, only one small study ${ }^{12}$ has investigated whether the conclusions of noninferiority trials vary by method of analysis, and whether per-protocol and as-treated analyses are more conservative.

Finally, a proposed ethical prerequisite for the use of a noninferiority design states that the NT must have known advantages such as reduced cost, greater convenience, or fewer side effects to justify the randomization of patients to a therapy with unknown efficacy. ${ }^{13}$ This requirement is not met in some noninferiority trials, ${ }^{14,15}$ but we are not aware of any empirical investigation of the frequency with which this occurs.

Our aim was to investigate how the aforementioned characteristics of noninferiority trials affect their results and the validity of their conclusions. 


\section{Conclusion regarding NT}

1 Superior

2 Noninferior

3 Noninferior

4 Noninferior

5 Inconclusive

6 Inconclusive

7 Inconclusive

8 Inferior
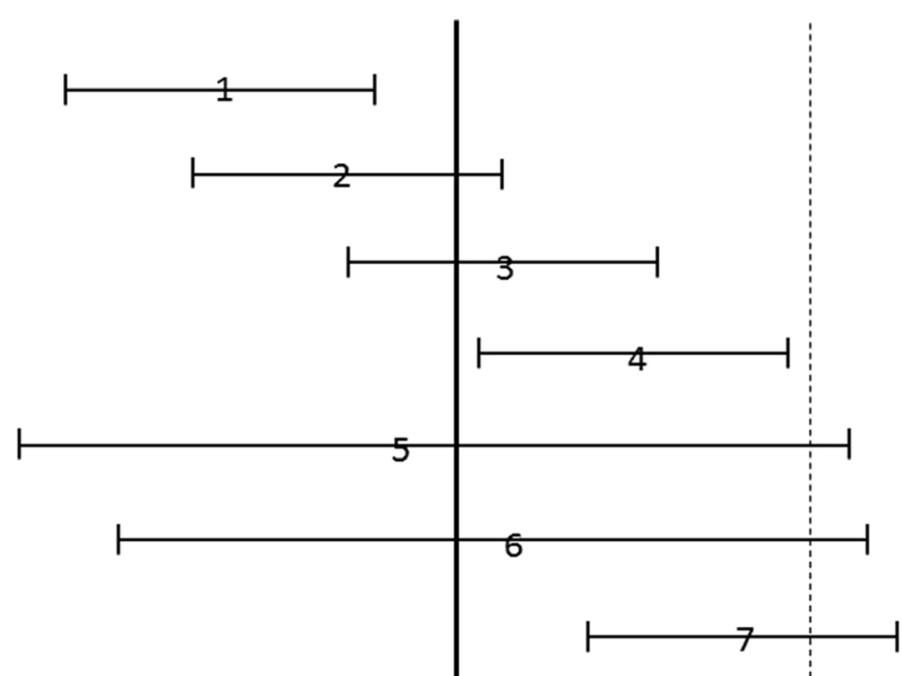



$\leftarrow$ New Treatment Better

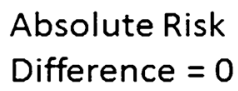

$\Delta$

Active Control Better $\rightarrow$

Figure 1 Simulacrum of the CONSORT diagram for interpreting the results of noninferiority trials. According to CONSORT, noninferiority can be declared whenever the upper bound of the confidence interval of the difference between the two therapies does not include delta, as in scenarios 1-4. Whenever the upper bound of the confidence interval exceeds delta, as in scenarios 5-7, noninferiority cannot be declared, because the plausible values of the parameter include some values greater than delta. When both the upper and lower bounds of the confidence interval exceed delta, the NT is declared inferior to the $\mathrm{AC}$, as in scenario 8. Scenario 1 represents all situations in which the upper bound of the confidence interval is less than zero - that is, any statistically significant result favoring the NT garners a declaration of superiority for the NT. By contrast, in scenarios 4 and 7, where there is a statistically significant difference favoring the AC, the NT is not declared inferior in this schematic, but rather noninferior (scenario 4) or inconclusive (scenario 7). NT, new treatment; AC, active control; $\Delta$, delta (the pre-specified margin of noninferiority).

\section{METHODS}

We searched MEDLINE using the search terms "noninferiority," "noninferior," "non-inferiority," and "non-inferior" combined with the name of the journals as recognized by MEDLINE and published between June 1, 2011, and November 1, 2016. We limited our search to the five highest-impact general medical journals in order to focus on the most widely cited and possibly highest-quality articles. ${ }^{16-18}$ We reviewed the resulting abstracts to identify articles that met our inclusion criteria: prospective, parallel-group randomized controlled trials where the primary outcome was tested using a noninferiority hypothesis. We then reviewed the full manuscripts and excluded trials that had a cluster-randomized design, trials where the data were incomplete or could not be summarized, those that used a Bayesian methodology, and those that did not use an AC (e.g., Food and Drug Administration [FDA]-mandated placebo-controlled post-approval safety studies). One author (SKA) abstracted the data from the trials into a standardized form and employed a system of redundant checks to ensure data accuracy. Another author (AMH) checked a sample of the data to ensure accuracy. We tabulated basic data about the trial including methodological and statistical design as reported. In trials where more than one analysis was reported (e.g., intention-to-treat [ITT], perprotocol [PP], as-treated [AT]), we determined whether the results differed depending on the analytical method.
We characterized trial results in terms of the point estimate and confidence intervals in accord with Figure 1 of the CONSORT statement, using a $95 \%$ two-sided confidence interval, calculated from the raw numbers provided in the manuscript, and coded the trial authors' conclusions regarding declarations of noninferiority, superiority, inferiority, or inconclusive results, and whether they differed from CONSORT. We used the first outcome mentioned in the manuscript as the primary outcome for our data set when multiple outcomes were reported. Some trials compared multiple interventions, e.g., multiple doses of a new drug against one AC group, and we considered these to represent separate comparisons. In determining whether justification for the selection of a delta value was presented, we coded trials as having "none" if no mention whatsoever was made as to how it was selected, "abstract" if some mention was made but it was vague or irreproducible, and "concrete" if an explicit reproducible justification was provided.

In determining whether the NT had advantages that justified its evaluation using a noninferiority design, we coded trials as having "none" if no advantages were mentioned nor could they be inferred, "inferred" if none were reported but they could be inferred, and "explicit" if the purported advantages of the NT were explicitly stated in the manuscript. Because we were interested in the numerical statistical design features of 
the trials, we did not perform a subjective quality analysis as is done in a traditional meta-analysis. Likewise, because of highly variable reporting of sponsor and funding sources, and the investigators' independence from them or lack thereof, we did not abstract these data.

Summary statistics are presented for abstracted values. Chisquare and Student's $t$ tests were used for exploratory analyses. Stata version 14 software (StataCorp LP, College Station, TX, USA) was used for all analyses, including recalculation of two-sided $95 \%$ confidence intervals.

\section{RESULTS}

Figure 2 shows the results of our search; 160 included manuscripts reported the results of 163 distinct trials and 182 noninferiority comparisons reported for those trials.

Table 1 shows the characteristics of the trials. The majority of trials (78\%) were published in the two highest-impact journals. Therapies pertaining to five specialties (infectious diseases, hematology/oncology, cardiology, pulmonary/ critical care, and endocrinology) accounted for $91 \%$ of all trials in our cohort. Reporting was complete (100\%), with no missing data for the primary outcome, delta, power, alpha sidedness, and planned sample size. Reporting of alpha was complete in $98.5 \%$ of trials, and the primary method of analysis was reported in $95.5 \%$. Justification for delta, the prespecified margin of noninferiority, was not reported in 58\% of trials, and in only $25 \%$ was reported in a way that allowed replicability of its method of determination. Almost one-third of the trials $(32 \%)$ used a two-sided alpha equivalent greater

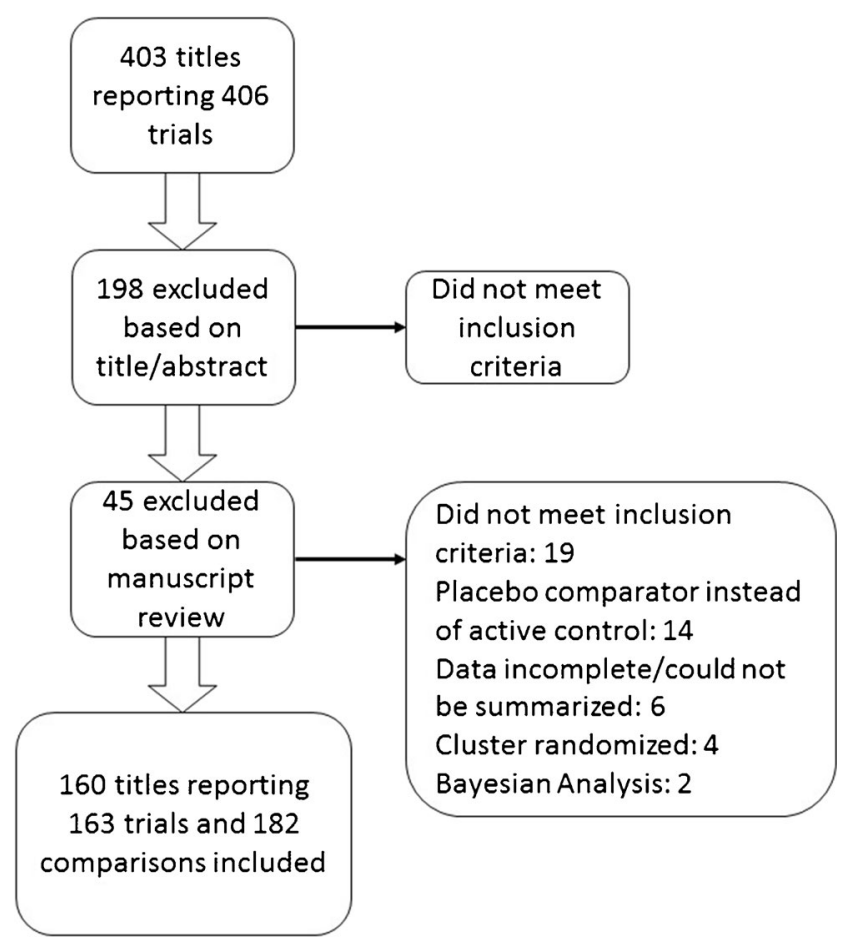

Figure 2 Flow diagram showing the results of our search. than the conventional standard for superiority trials $(0.05)$, with four trials using the equivalent of a two-sided alpha of 0.20 .

For trials that used primary outcomes convertible to an absolute risk difference $(n=137)$, the mean pre-specified delta was $8.7 \%$, with a range of $0.4 \%$ to $25 \%$. For trials where mortality was the primary outcome or part thereof $(n=48)$, the mean pre-specified delta was $6.1 \%$, with a range of $0.4 \%$ to $19.1 \%$, and if mortality was not part of the outcome $(n=89)$, the mean pre-specified delta was $10.0 \%$, with a range of $0.57 \%$ to $25 \%$. The mean observed delta for the primary outcome in the 151 comparisons convertible to an absolute risk difference was $+0.04 \%$ (range $-36.6 \%$ to $+26.9 \%$ ), with 61 point estimates favoring NT and 63 favoring AC. Of the total 182 comparisons, 95 point estimates favored the new treatment and 87 favored the alternative.

Among all 182 comparisons, 28 (15\%) were categorized as demonstrating the new treatment to be superior (scenario 1); $113(62 \%)$ were classified as noninferior (scenario 2 or 3); three $(2 \%)$ were found to show statistically significant evidence that the new treatment was worse but were considered noninferior (scenario 4); 19 (10\%) were inconclusive (scenario 5 or 6$)$; in $15(8 \%)$ the new treatment was statistically significantly worse, but the result was considered inconclusive (scenario 7); and four (2.0\%) found the new treatment to be inferior (scenario 8 ). In total, there were 28 statistically significant results favoring the NT (scenario 1), and 22 statistically significant results favoring the comparator therapy (scenarios 4, 7, and 8 combined; difference not significant). Table 2 lists the trials where a scenario 4 or 7 result was obtained.

Figure 3 shows the log of the number of patients analyzed in each trial plotted against the observed result as an absolute risk difference (ARD) for 151 comparisons where an ARD could be calculated. Our results, following CONSORT recommendations, are color coded such that blue dots represent noninferiority, green superiority, yellow inconclusive results, and black inferiority of the NT. There is a paucity of data points on the bottom right of the figure where small trials show large differences favoring the $\mathrm{AC}$, a finding that suggests publication bias. However, formal tests of publication bias ( $\mathrm{Begg}^{36}$ and Harbord ${ }^{37}$ ), which are known to be insensitive, were not statistically significant. Figure 4 shows the same data, but here, statistically significant differences favoring AC which were previously coded as inconclusive (CONSORT 7) or noninferior (CONSORT 4) are coded as red. Figure 4 shows that there is a nearly symmetrical distribution of point estimates around a difference of zero. In this figure, there are 23 green data points, and a combined 19 red and black data points, representing a similar distribution of statistically significant results favoring NT and AC, respectively.

In five of the comparisons where the authors used an alpha threshold less stringent than a two-sided 0.05 equivalent, our use of a two-sided $95 \%$ confidence interval changed the conclusions. In each of these cases, the more stringent confidence interval made the result less favorable for the NT. In one trial, use of a two-sided $95 \%$ confidence interval changed the result from superior to noninferior. ${ }^{38}$ In three trials, ${ }^{20,24,25}$ the use of 
Table 1 Characteristics of the Trials in Our Cohort

\begin{tabular}{|c|c|c|}
\hline & & No. (\%) \\
\hline \multirow[t]{5}{*}{ Journal } & NEJM & $64(39 \%)$ \\
\hline & Lancet & $63(39 \%)$ \\
\hline & JAMA & $23(14 \%)$ \\
\hline & BMJ & $8(5 \%)$ \\
\hline & Annals & $5(3 \%)$ \\
\hline \multirow[t]{6}{*}{ Year } & June-December 2011 & $12(7 \%)$ \\
\hline & 2012 & $25(15 \%)$ \\
\hline & 2013 & $34(21 \%)$ \\
\hline & 2014 & $22(14 \%)$ \\
\hline & 2015 & $43(26 \%)$ \\
\hline & January-October 2016 & $27(17 \%)$ \\
\hline \multirow[t]{5}{*}{ Top specialties } & Infectious diseases & $26 \%$ \\
\hline & Hematology/oncology & $25 \%$ \\
\hline & Cardiology & $17 \%$ \\
\hline & Pulmonary/critical care & $15 \%$ \\
\hline & Endocrinology & $8 \%$ \\
\hline \multirow[t]{5}{*}{ Primary outcome measure ( $n=163$ trials) } & Absolute risk difference & $114(70 \%)$ \\
\hline & Mean & $26(16 \%)$ \\
\hline & Hazard ratio & $13(8 \%)$ \\
\hline & Relative risk difference & $8(5 \%)$ \\
\hline & Odds ratio & $2(1 \%)$ \\
\hline Primary analysis & Intention-to-treat & $95(58 \%)$ \\
\hline \multirow[t]{4}{*}{$(n=163$ trials $)$} & Modified intention-to-treat & $36(22 \%)$ \\
\hline & Per-protocol & $24(15 \%)$ \\
\hline & As treated & $2(1 \%)$ \\
\hline & Not reported & $6(4 \%)$ \\
\hline Secondary analysis & Intention-to-treat & $14(9 \%)$ \\
\hline \multirow{4}{*}{$(n=163$ trials $)$} & Modified intention-to-treat & $7(4 \%)$ \\
\hline & Per-protocol & $79(50 \%)$ \\
\hline & As treated & $10(6 \%)$ \\
\hline & None & $48(30 \%)$ \\
\hline Outcome is or includes mortality ( $n=163$ trials) & & $49(30 \%)$ \\
\hline \multirow{9}{*}{$\begin{array}{l}\text { Delta for comparisons with proportional outcome } \\
\text { measure ( } n=137 \text { trials) }\end{array}$} & Mean delta & 0.087 \\
\hline & Lowest value & 0.004 \\
\hline & Highest value & 0.25 \\
\hline & $\begin{array}{l}\text { Mean delta for outcomes that do not include } \\
\text { mortality }(n=89)\end{array}$ & 0.1 \\
\hline & Lowest value for outcomes that do not include mortality & 0.0057 \\
\hline & Highest value for outcomes that do not include mortality & 0.25 \\
\hline & Mean delta for outcomes that include mortality $(n=48)$ & 0.061 \\
\hline & Lowest value for outcomes that include mortality & 0.004 \\
\hline & Highest value for outcomes that include mortality & 0.19 \\
\hline \multirow[t]{3}{*}{ Delta justification ( $n=163$ trials) } & Not reported & $95(58 \%)$ \\
\hline & Vague and non-reproducible & $27(17 \%)$ \\
\hline & Concrete and reproducible & $41(25 \%)$ \\
\hline \multirow[t]{4}{*}{ Alpha, one-sided equivalent ( $n=163$ trials) } & $\leq 0.025$ & $105(66 \%)$ \\
\hline & $\overline{0} .05$ & $51(32 \%)$ \\
\hline & 0.1 & $4(2.5 \%)$ \\
\hline & Not reported & $3(2 \%)$ \\
\hline Two-sided hypothesis test ( $n=163$ trials $)$ & & $62(38 \%)$ \\
\hline \multirow{8}{*}{$\begin{array}{l}\text { CONSORT confidence interval categorization } \\
(n=183 \text { comparisons })\end{array}$} & 1 (new treatment superior) & $28(15 \%)$ \\
\hline & 2 (new treatment noninferior) & $67(37 \%)$ \\
\hline & 3 (new treatment noninferior) & $46(25 \%)$ \\
\hline & $\begin{array}{l}4 \text { (new treatment noninferior, but old treatment } \\
\text { statistically better by less than delta) }\end{array}$ & $3(2 \%)$ \\
\hline & 5 (inconclusive) & $0(0 \%)$ \\
\hline & 6 (inconclusive) & $19(10 \%)$ \\
\hline & $\begin{array}{l}7 \text { (inconclusive, but old treatment statistically better, } \\
\text { by less than delta) }\end{array}$ & $15(8 \%)$ \\
\hline & 8 (new treatment inferior) & $4(2 \%)$ \\
\hline \multirow[t]{3}{*}{ Advantage of new therapy } & Explicitly stated & $114(70 \%)$ \\
\hline & Could be inferred & $31(19 \%)$ \\
\hline & Neither stated nor able to be inferred & $18(11 \%)$ \\
\hline
\end{tabular}

NEJM, New England Journal of Medicine; Annals, Annals of Internal Medicine

a two-sided $95 \%$ confidence interval caused a change in the classification of the result from scenario 6 (inconclusive) to scenario 7 (the NT is statistically significantly worse but the result is deemed inconclusive), and in one trial ${ }^{39}$ it changed a scenario 3 (noninferior) to scenario 7 (inconclusive). In all these instances, it was the change from a one-sided to a twosided confidence interval that changed the categorization of the result, rather than increased stringency from the use of a smaller one-sided alpha value. In the most extreme case (antibiotics vs. appendectomy for acute appendicitis), ${ }^{25}$ the use of a one-sided confidence interval concealed a large and highly statistically significant result in favor of the AC (absolute difference $=27 \% ; 95 \%$ CI 21.3-32.9\%). Two other trials had statistically significant results favoring the AC by $7 \%$ or 
Table 2 Trials with Statistically Significant Results Disfavoring the New Therapy but Not Considering It Inferior

\begin{tabular}{|c|c|c|c|c|c|c|}
\hline First author & Disease & New therapy & Active control & Outcome & Delta & Result (95\% CI) \\
\hline Roberts $^{19}$ & $\begin{array}{l}\text { Pediatric } \\
\text { respiratory failure }\end{array}$ & $\begin{array}{l}\text { High-flow nasal } \\
\text { cannula }\end{array}$ & CPAP & Tx failure & 0.1 & $0.122(0.06-0.19)$ \\
\hline Geisler $^{20}$ & Chlamydia infection & Azithromycin & Doxycycline & Tx failure & 0.05 & $0.032(0.004-0.06)$ \\
\hline $\mathrm{Kaul}^{21} *$ & Coronary disease & Paclitaxel stent & Everolimus stent & $\begin{array}{l}\text { Composite, } \\
\text { including death }\end{array}$ & 0.04 & $0.027(0.01-0.05)$ \\
\hline Gillespie $^{22}$ & Tuberculosis & $\begin{array}{l}\text { Moxifloxacin } \\
\text { replaces } \\
\text { ethambutol }\end{array}$ & Standard TB Tx & Tx failure & 0.06 & $0.07(0.03-0.11)$ \\
\hline $\begin{array}{l}\text { Bwakura- } \\
\text { Danbarembizi }\end{array}$ & HIV & $\begin{array}{l}\text { Stopping SMX } \\
\text { prophylaxis }\end{array}$ & $\begin{array}{l}\text { Continued SMX } \\
\text { prophylaxis }\end{array}$ & $\begin{array}{l}\text { Hospitalization } \\
\text { or death }\end{array}$ & 0.03 & $0.06(0.01-0.11)$ \\
\hline Stevenson ${ }^{24}$ & Rectal cancer & $\begin{array}{l}\text { Laparoscopic } \\
\text { surgery }\end{array}$ & Open surgery & $\begin{array}{l}\text { Pathological } \\
\text { outcomes }\end{array}$ & 0.08 & $0.07(0.01-0.13)$ \\
\hline Salminen ${ }^{25 \dagger}$ & Appendicitis & Antibiotics & Surgery & Tx failure & 0.24 & $0.27(0.22-0.33)$ \\
\hline Hooton $^{26}$ & $\begin{array}{l}\text { Urinary tract } \\
\text { infection }\end{array}$ & Cefpodoxime & Ciprofloxacin & Clinical cure & 0.1 & $0.11(0.03-0.18)$ \\
\hline Bachelez $^{27}$ & $\begin{array}{l}\text { Rheumatoid } \\
\text { arthritis }\end{array}$ & $\begin{array}{l}\text { Tofacitinib } \\
5 \mathrm{mg} \text { dose }\end{array}$ & Etanercept & PASI75 score & 0.15 & $0.19(0.12-0.27)$ \\
\hline Behringer $^{28}$ & Hodgkin's lymphoma & $\mathrm{ABV}$ & ABVD & Tx failure & 0.06 & $0.12(0.06-0.18)$ \\
\hline Behringer $^{28}$ & $\begin{array}{l}\text { Hodgkin's } \\
\text { lymphoma }\end{array}$ & AVD & $\mathrm{ABVD}$ & Tx failure & 0.06 & $0.04(0.01-0.07)$ \\
\hline Vaidya $^{29}$ & Breast cancer & Targeted XRT & Whole breast XRT & Local recurrence & 0.025 & $0.007(0.0004-0.014)$ \\
\hline Buse $^{30}$ & Diabetes & Exenatide & Liraglutide & $\begin{array}{l}\text { Mean change in } \\
\text { glycated hemoglobin }\end{array}$ & $0.25 \%$ & $0.0021(0.0008-0.0033)$ \\
\hline $\begin{array}{l}\text { Lindson- } \\
\text { Hawley }^{31}\end{array}$ & Smoking & Gradual cessation & Abrupt cessation & Smoking cessation & 0.095 & $0.10(0.03-0.17)$ \\
\hline Perkins ${ }^{32}$ & ACLS training & E-learning & In-person training & Pass rate & 0.05 & $0.06(0.03-0.09)$ \\
\hline $\mathrm{Mol}^{33}$ & Venous thrombosis & 1-year TED hose & 2-year TED hose & $\begin{array}{l}\text { Post-thrombotic } \\
\text { syndrome rate }\end{array}$ & 0.1 & $0.07(0.01-0.13)$ \\
\hline Gallwitz $^{34 \$}$ & Diabetes & Linagliptin & Glimepiride & $\begin{array}{l}\text { Mean change } \\
\text { in glycated } \\
\text { hemoglobin }\end{array}$ & $0.35 \%$ & $0.2(0.09-0.30)$ \\
\hline Fishbane $^{35 \ddagger}$ & Renal failure & Peginesatide & Epoetin & $\begin{array}{l}\text { Mean change in } \\
\text { hemoglobin }\end{array}$ & 1 & $0.15(0.01-0.30)$ \\
\hline
\end{tabular}

These trials are categorized as scenarios 4 and 7 based on the Figure 1 diagram. Abbreviations: Tx, treatment; CPAP, continuous positive airway pressure; XRT, X-ray therapy; SMX, sulfamethoxazole; ACLS, advanced cardiac life support

*The authors state in the introduction that paclitaxel stents are inferior to everolimus stents in most patients; in an apparent post hoc analysis, they reverse the noninferiority hypothesis and declare everolimus stents superior to paclitaxel stents, a conclusion that they acknowledged was known before the trial was conducted

"Use of a one-sided 95\% CI with an upper bound of 0.32 allowed the authors to correctly classify the result as "inconclusive"

* One of three CONSORT 4 results where the entire 95\% CI lies between 0 and $\Delta$, and the result is considered noninferior

more that were concealed by the CONSORT interpretative framework.

For eight comparisons, the authors reported conclusions that differed materially from the categorizations recommended

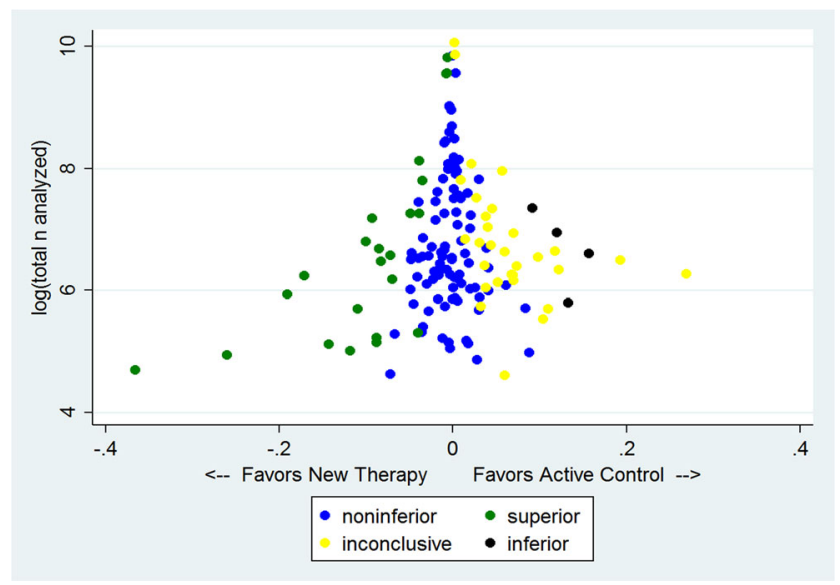

Figure 3 Plot of 151 comparisons of absolute risk differences as a function of the log of the total number of patients analyzed in the trial, color coded by the interpretation of the results as recommended by CONSORT. See text for details. by CONSORT. In two trials, ${ }^{40,}{ }^{41}$ a CONSORT 1 result was obtained, but the authors concluded noninferiority rather than superiority. In two trials, ${ }^{22,} 28$ the authors reported that "noninferiority was not shown" rather than "inferiority was

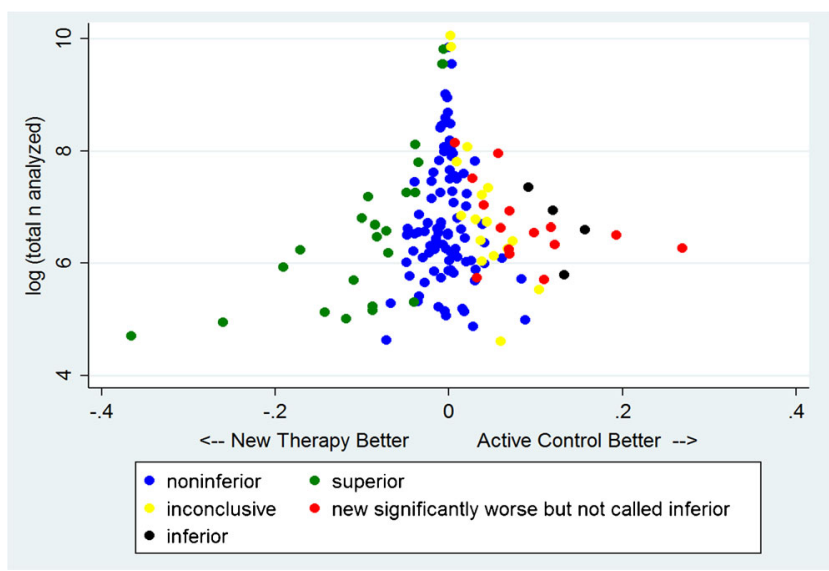

Figure 4 Plot of 151 comparisons with a calculable absolute risk difference as in Figure 3, but with statistically significant results in favor of active control (AC), coded as inconclusive or noninferior in Figure 3, denoted by red in Figure 4. 
shown." In three trials where a CONSORT 7 result was obtained (the NT was statistically significantly worse but the result deemed inconclusive), the authors reported that the new treatment was inferior, ${ }^{42}$ stated that the new treatment was statistically worse without concluding inferiority, ${ }^{19}$ or reversed the noninferiority hypothesis and declared the $\mathrm{AC}$ superior to the NT. ${ }^{21}$ All eight instances where there was a material difference between the conclusions reported and those recommended by CONSORT involved a statistically significant difference in favor of either AC or NT.

In five of the 117 comparisons where more than one analysis method was reported (e.g., ITT and PP), the conclusion differed depending upon the analysis method. In four ${ }^{42-45}$ comparisons, the secondary PP analysis was less conservative than the primary analysis, i.e., noninferiority could be claimed with the PP analysis but not the primary analysis. In one trial, ${ }^{46}$ the secondary PP analysis was more conservative and noninferiority criteria were met only with the primary ITT analysis. The authors claimed noninferiority despite this discrepancy. ${ }^{46}$

Among all 163 trials, $70 \%$ concretely stated the purported advantage of the NT which would provide ethical justification for randomization with a noninferiority design. In $19 \%$ of trials, the advantage was not stated but could be inferred by the reviewers, and in $11 \%$ of trials, the advantage of the NT was not stated nor could it be inferred. For more than half of the latter, the NT was a cardiovascular stent device. ${ }^{15}$

\section{DISCUSSION}

In our study of 163 noninferiority trials with 182 noninferiority comparisons published in the five highest-impact general medicine journals during a recent 5 -year period, we found that current interpretive recommendations lead to significant systematic and directional biases in the analysis and interpretation of noninferiority trials which almost always favor the experimental therapy. To our knowledge, our analysis is the first to abstract raw data, calculate confidence intervals, categorize the results based on CONSORT, and explore whether and how deviation from the customary design parameters of superiority trials impacts the results and conclusions of noninferiority trials. This is important, since decisions about the use of new, often more expensive therapies increasingly rely on data from noninferiority trials. Most previous analyses of such trials have focused on quality and completeness of reporting of design parameters and results. ${ }^{17,}{ }^{47} \mathrm{We}$ found that statistical design parameters (excluding justification for delta which is perennially deficient) and results were reported with near $100 \%$ completeness in the period we studied. However, despite nearly complete reporting, we found that the interpretation of the results of noninferiority trials can hinge critically on both the choice of design parameters and the method of drawing formal conclusions from the results. In our cohort, the use of CONSORT-recommended interpretation with $95 \%$ confidence intervals concealed statistically significant results that disfavored the NT in a substantial number of cases. The use of one-sided confidence intervals also concealed statistically significant results disfavoring the NT in a small number of comparisons. Contrary to the recommended preferential use of PP or AT analyses over ITT analyses, we found that the method of analysis seldom affected the results, and when it did, the ITT analysis was more conservative in four out of five trials. While the issues we describe each affect a minority of the trials we analyzed, the collective effect is substantial.

One of us previously suggested that the use of the CONSORT diagram for the interpretation of the results of noninferiority trials could result in bias. ${ }^{7}$ Our results empirically demonstrate that an asymmetrical interpretation of noninferiority trials creates bias that favors the NT, as illustrated in Figure 5. This figure shows the same results as Figure 1, but as a mirror image, with the AC on the left and the NT on the right of the diagram. As described in the caption, reversing the designation in this way results in a material change to the conclusions in four of the eight scenarios, making the results less favorable for NT. While it has been argued that the designation of NT and AC are not arbitrary because the NT is chronologically "new," 48 we found cases in our cohort where the NT was a pre-existing or "old" therapy. ${ }^{49,}{ }^{50}$ In other trials, placebo was assigned NT status and compared to an unproven treatment as $\mathrm{AC},{ }^{51-56}$ and in 31 trials, therapies at full intensity were assigned as $\mathrm{AC}$ and compared to the same therapies at reduced intensity assigned as NT (Aberegg et al., manuscript under review). Thus, chronology alone appears to be an insufficient criterion for assignment to preferred status as NT. Our results provide the first empirical evidence that this method of interpretation is biased in favor of NT and, in a nontrivial number of trials, effects the concealment of statistically significant results disfavoring NT within the interpretive framework.

Numerous previous reports have examined the adequacy of reporting of noninferiority trials, and have focused consistently on the methodology for the selection of the delta margin. ${ }^{17}$, ${ }^{47},{ }^{57}$ Le Henanff et al. ${ }^{47}$ (2006) reported significant deficiencies in the reporting of statistical design parameters, justification for the delta margin selected (only $20 \%$ of reports in their cohort), and reporting of analysis method, with only $43 \%$ reporting more than one method (e.g., ITT and PP). Wangge et al. ${ }^{57}$ (2010) found that justification for the delta margin had increased to $46 \%$, but the percentage reporting both types of analysis had not changed. In 2016, Rehal et al. ${ }^{17}$ found that justification for the delta margin remained stubbornly anchored at $46 \%$, with multiple methods of analysis reported in $54 \%$. These reports also described the trial authors' selections regarding alpha and its sidedness; however, our report is the first to describe and quantify the empirical consequences of these selections. Other investigators have noted the potential advantages of preferential use of ITT analyses in noninferiority trials, ${ }^{4,9,10}$ but the only other empirical analysis of the ITT/PP difference included just 20 trials and found results that 


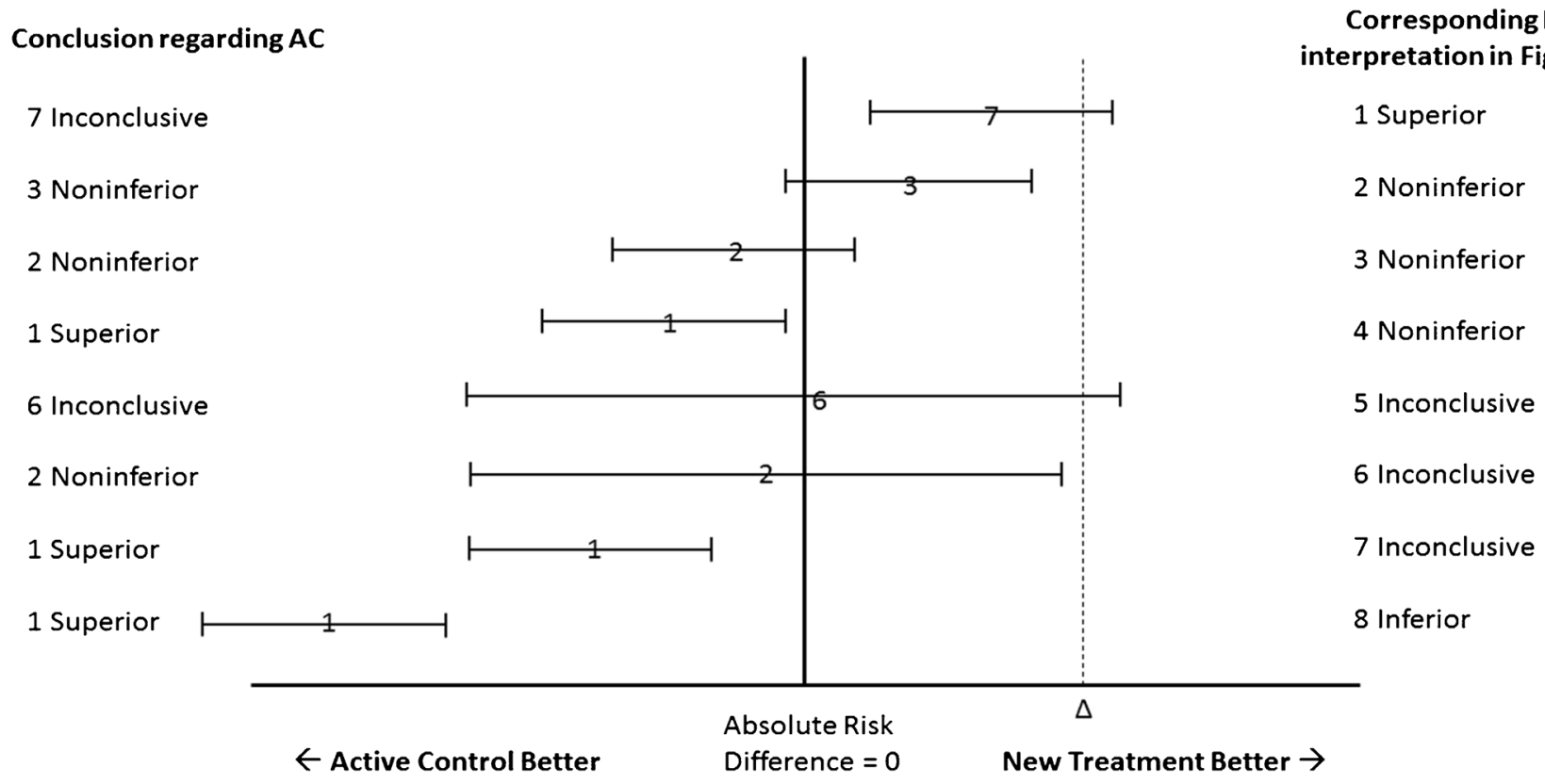

Figure 5 The effect of asymmetrical interpretation of noninferiority results. This schematic shows how the conclusions of a noninferiority trial will differ depending upon which agent, NT or AC, is assigned preferential status on the left of the interpretative diagram. The confidence intervals in this diagram are the mirror images of the confidence intervals in Figure 1, but the NT is now on the right, and AC is on the left (favored side) of the diagram. The absolute risk differences between NT and AC are the same as in Figure 1, and the diagram represents the interpretation that would result if the hypothesis were set up in reverse, to test the noninferiority of AC to NT. The top confidence interval shows a statistically significant difference favoring the new treatment, but instead of a conclusion of superiority (designation 1 in Fig. 1), the result is inconclusive because the upper bound of the confidence interval crosses delta. For the second and third confidence intervals, the conclusion of noninferiority does not change. For the fourth confidence interval, the prior designation 4 (NT is noninferior to AC) becomes a designation 1 (AC superior to NT). The designation of the fifth confidence interval does not change, but the sixth, previously designated inconclusive, becomes a noninferior result for $\mathrm{AC}$. The seventh and eighth confidence intervals, previously showing inconclusive and inferior results for NT, are now designated superior results for AC. Note that the experimental results have not changed-only the assignment of one agent to preferential status on the left of the diagram. Among the eight confidence intervals, four conclusions are materially changed when preferential status is changed.

comport with ours, with the ITT analysis being more conservative. ${ }^{12}$

We found that point estimates from trials in our cohort were nearly symmetrically distributed around a difference of zero, similar to two prior investigations. ${ }^{18,58}$ A large analysis of superiority trials found a similar symmetrical distribution in new versus established treatments. ${ }^{59}$ While the stochastic nature of these results is of epistemological interest and open to speculative interpretation, we reason that this pattern provides support for the idea that new treatments, on average, are not substantially better than existing treatments. On this basis alone, the allowance of preferential treatment for NT is unjustified, especially given our finding that for many NTs, a specific secondary advantage of the NT was not stated and could not be inferred. ${ }^{14,15}$

Our results have significant implications for the design and analysis of future noninferiority trials. The use of the CONSORT diagram (Fig. 1) for the classification of results should be reevaluated given its obfuscation of results disfavoring NT by a statistically significant margin in $12 \%$ of trials in our cohort. This view is bolstered by the fact that the authors of these reports had various ways of drawing conclusions in these CONSORT 4 and 7 scenarios, and that their conclusions often differed from the recommendations. An alternative approach would be to conclude superiority of any treatment with a statistically significant difference favoring it regardless of direction or size. This would remedy much of the directional bias resulting from asymmetry seen in Figure 5. Alternatively, the noninferiority design could be abandoned in favor of equivalence trials with symmetrical delta margins on both sides of unity. ${ }^{4,60}$

Our finding that there are rarely differences between ITT and PP analyses, and that ITT is usually more conservative when differences arise, suggests that the recommendation to preferentially use PP or AT analyses, which can defeat randomization, should be reevaluated. $4,9,10$ Our results also confirm that one-sided confidence intervals can sometimes conceal large $\mathrm{e}^{25}$ and statistically significant differences in outcomes disfavoring the NT. Universal use of two-sided confidence intervals would be preferable. Finally, in addition to an explicit statement justifying the choice of delta, authors of these reports should be required to make an explicit statement about the purported secondary advantages of the NT that form the ethical basis for randomization to an unproven therapy when an effective therapy exists. ${ }^{3,13}$

Some of the proposed changes to noninferiority trials, such as use of equivalence designs, two-sided confidence intervals, and conservative delta margins, will put upward pressure on sample size in these trials. The implementation of one change could therefore put pressure on another variable upon which 
sample size depends. Thus, we may expect that the use of more conservative delta values and two-sided confidence intervals could cause investigators to use more lax alpha values or relax power in order to maintain constant sample size. ${ }^{16}$ These compensations could have downstream impacts on the results of future trials. Therefore, it may be most prudent to recommend standardization of statistical design parameters, as is done with superiority trials, and require the uniform use of two-sided $95 \%$ confidence intervals in addition to an explicit justification for delta. This standardization would also remove a "researcher degree of freedom" ${ }^{\prime 61}$ in the analysis of the results - a very important consideration since pre-registration of noninferiority trials on www.clinicaltrials.gov generally does not include data about statistical design parameters.

A major strength of the present work is that it was a hypothesis-driven $^{7}$ descriptive study to evaluate the empirical consequences of several design recommendations within a theoretical framework. We surveyed a substantial publication epoch in the five highest-impact general medical journals which should capture important noninferiority trials published during that time. Our analysis targeted empirical findings that have not heretofore been reported in analyses of noninferiority trials. Limitations include the selection of only five journals over a 5.5 -year period to make our data set manageable, given the labor-intensive nature of our data extraction and recalculation of $95 \%$ confidence intervals. Future studies could attempt to confirm our results in a broader selection of journals and over an expanded date range. The possibility of publication bias may influence the results, but our data suggest that publication bias, if present, primarily affects small trials with results favoring AC, and if this is true, our results may understate problems with these trials. Limiting our analysis to the five highest-impact general medical journals may impact the generalizability of our results, but we reason that the included journals likely publish trials of the highest methodological quality, which would cause our results to be an understatement of problems with noninferiority trials.

\section{CONCLUSIONS}

The current design and interpretation of noninferiority trials can lead to conclusions which are biased in favor of the therapy designated as new. Clinicians utilizing this literature should cautiously inspect the actual results rather than rely on authors' interpretations and conclusions. Future noninferiority trials may benefit from standardized design parameters, as is currently customary with superiority trials.

Corresponding Author: Scott K. Aberegg, MD, MPH; Pulmonary Division, University of Utah School of Medicine, $30 \mathrm{~N} 1900$ E, 701 Wintrobe, Salt Lake City, UT 84132, USA (e-mail: scottaberegg@gmail. com).

\section{Compliance with Ethical Standards:}

Conflict of Interest: The authors declare that they have no conflict of interest.
Open Access This article is distributed under the terms of the Creative Commons Attribution 4.0 International License (http:// creativecommons.org/licenses/by/4.0/), which permits unrestricted use, distribution, and reproduction in any medium, provided you give appropriate credit to the original author(s) and the source, provide a link to the Creative Commons license, and indicate if changes were made.

\section{REFERENCES}

1. Murthy VL, Desai NR, Vora A, Bhatt DL. Increasing proportion of clinical trials using noninferiority end points. Clin Cardiol. 2012;35(9):522-523.

2. Suda KJ, Hurley AM, McKibbin T, Motl Moroney SE. Publication of noninferiority clinical trials: changes over a 20-year interval. Pharmacotherapy. 2011;31(9):833-839.

3. D'Agostino RB, Sr., Massaro JM, Sullivan LM. Non-inferiority trials: design concepts and issues - the encounters of academic consultants in statistics. Stat Med. 2003;22(2):169-186.

4. Fleming TR. Current issues in non-inferiority trials. Stat Med. 2008;27(3):317-332.

5. Piaggio G, Elbourne DR, Altman DG, Pocock SJ, Evans SJ. Reporting of noninferiority and equivalence randomized trials: an extension of the CONSORT statement. JAMA. 2006;295(10):1152-1160.

6. Piaggio G, Elbourne DR, Pocock SJ, Evans SW, Altman DG, for the CONSORT Group. Reporting of noninferiority and equivalence randomized trials: extension of the CONSORT 2010 statement. JAMA. 2012;308(24):2594-2604.

7. Aberegg S. Reporting noninferiority trials. JAMA. 2013;309(15):15841585.

8. Matsuyama Y. A comparison of the results of intent-to-treat, perprotocol, and g-estimation in the presence of non-random treatment changes in a time-to-event non-inferiority trial. Stat Med. 2010;29(20):2107-2116.

9. Wiens BL, Zhao W. The role of intention to treat in analysis of noninferiority studies. Clin Trials. 2007;4(3):286-291.

10. Sheng $\mathbf{D}$, Kim MY. The effects of non-compliance on intent-to-treat analysis of equivalence trials. Stat Med. 2006;25(7):1183-1199.

11. Matilde Sanchez M, Chen $\mathbf{X}$. Choosing the analysis population in noninferiority studies: per protocol or intent-to-treat. Stat Med. 2006;25(7):1169-1181.

12. Brittain E, Lin D. A comparison of intent-to-treat and per-protocol results in antibiotic non-inferiority trials. Stat Med. 2005;24(1):1-10.

13. Garattini S, Bertele V. Non-inferiority trials are unethical because they disregard patients' interests. Lancet. 2007;370(9602):18751877.

14. Ardehali A, Esmailian F, Deng M, et al. Ex-vivo perfusion of donor hearts for human heart transplantation (PROCEED II): a prospective, open-label, multicentre, randomised non-inferiority trial. Lancet. 2015;385(9987):2577-2584.

15. Byrne RA, Kastrati A. Drug-eluting stent trials: too much noninferiority, too little progress? Lancet. 2014;383(9915):386-388.

16. Aberegg SK, Richards DR, O'Brien JM. Delta inflation: a bias in the design of randomized controlled trials in critical care medicine. Crit Care. 2010;14(2):R77.

17. Rehal S, Morris TP, Fielding $\mathbf{K}$, Carpenter JR, Phillips PPJ. Noninferiority trials: are they inferior? A systematic review of reporting in major medical journals. BMJ Open. 2016;6(10).

18. Soonawala D, Middelburg RA, Egger M, Vandenbroucke JP, Dekkers OM. Efficacy of experimental treatments compared with standard treatments in non-inferiority trials: a meta-analysis of randomized controlled trials. Int $\mathrm{J}$ Epidemiol. 2010;39(6):15671581.

19. Roberts CT, Owen LS, Manley BJ, et al. Nasal high-flow therapy for primary respiratory support in preterm infants. N Engl J Med. 2016;375(12):1142-1151.

20. Geisler WM, Uniyal A, Lee JY, et al. Azithromycin versus doxycycline for urogenital chlamydia trachomatis infection. N Engl J Med. 2015;373(26):2512-2521.

21. Kaul U, Bangalore S, Seth A, et al. Paclitaxel-eluting versus everolimuseluting coronary stents in diabetes. N Engl J Med. 2015;373(18):17091719.

22. Gillespie SH, Crook AM, McHugh TD, et al. Four-month moxifloxacinbased regimens for drug-sensitive tuberculosis. N Engl J Med. 2014;371(17):1577-1587. 
23. Bwakura-Dangarembizi M, Kendall L, Bakeera-Kitaka S, et al. A randomized trial of prolonged Co-trimoxazole in HIV-infected children in Africa. N Engl J Med. 2014;370(1):41-53.

24. Stevenson AL, Solomon MJ, Lumley JW, et al. Effect of laparoscopicassisted resection vs open resection on pathological outcomes in rectal cancer: the ALaCaRT randomized clinical trial. JAMA. 2015;314(13): 1356-1363.

25. Salminen $\mathbf{P}$, Paajanen $\mathbf{H}$, Rautio $\mathbf{T}$, et al. Antibiotic therapy vs appendectomy for treatment of uncomplicated acute appendicitis: the APPAC randomized clinical trial. JAMA. 2015;313(23):2340-2348.

26. Hooton TM, Roberts PL, Stapleton AE. Cefpodoxime vs ciprofloxacin for short-course treatment of acute uncomplicated cystitis: a randomized trial. JAMA. 2012;307(6):583-589.

27. Bachelez H, van de Kerkhof PCM, Strohal R, et al. Tofacitinib versus etanercept or placebo in moderate-to-severe chronic plaque psoriasis: a phase 3 randomised non-inferiority trial. Lancet. 2015;386(9993):552561.

28. Behringer $\mathbf{K}$, Goergen $\mathbf{H}$, Hitz $\mathbf{F}$, et al. Omission of dacarbazine or bleomycin, or both, from the ABVD regimen in treatment of early-stage favourable Hodgkin's lymphoma (GHSG HD13): an open-label, randomised, non-inferiority trial. Lancet. 2015;385(9976):1418-1427.

29. Vaidya JS, Wenz F, Bulsara M, et al. Risk-adapted targeted intraoperative radiotherapy versus whole-breast radiotherapy for breast cancer: 5-year results for local control and overall survival from the TARGIT-A randomised trial. Lancet. 2014;383(9917):603-613.

30. Buse JB, Nauck M, Forst T, et al. Exenatide once weekly versus liraglutide once daily in patients with type 2 diabetes (DURATION-6): a randomised, open-label study. Lancet. 2013;381(9861):117-124.

31. Lindson-Hawley N, Banting M, West R, Michie S, Shinkins B, Aveyard P. Gradual versus abrupt smoking cessation: a randomized, controlled noninferiority trial. Ann Intern Med. 2016;164(9):585-592.

32. Perkins GD, Kimani PK, Bullock I, et al. Improving the efficiency of advanced life support training: a randomized, controlled trial. Ann Intern Med. 2012;157(1):19-28.

33. Mol GC, van de Ree MA, Klok FA, et al. One versus two years of elastic compression stockings for prevention of post-thrombotic syndrome (OCTAVIA study): randomised controlled trial. BMJ. 2016;353.

34. Gallwitz B, Rosenstock J, Rauch T, et al. 2-year efficacy and safety of linagliptin compared with glimepiride in patients with type 2 diabetes inadequately controlled on metformin: a randomised, double-blind, noninferiority trial. Lancet. 2012;380(9840):475-483.

35. Fishbane S, Schiller B, Locatelli F, et al. Peginesatide in patients with anemia undergoing hemodialysis. N Engl J Med. 2013;368(4):307-319.

36. Begg CB, Mazumdar M. Operating characteristics of a rank correlation test for publication bias. Biometrics. 1994;50(4):1088-1101.

37. Harbord RM, Egger M, Sterne JAC. A modified test for small-study effects in meta-analyses of controlled trials with binary endpoints. Stat Med. 2006;25(20):3443-3457.

38. Adams DH, Popma JJ, Reardon MJ, et al. Transcatheter aortic-valve replacement with a self-expanding prosthesis. N Engl J Med. 2014;370(19): 1790-1798.

39. Hernández G, Vaquero C, Colinas L, et al. Effect of postextubation highflow nasal cannula vs noninvasive ventilation on reintubation and postextubation respiratory failure in high-risk patients: a randomized clinical trial. JAMA. 2016;316(15):1565-1574.

40. Coovadia A, Abrams EJ, Strehlau R, et al. Efavirenz-based antiretroviral therapy among nevirapine-exposed HIV-infected children in South Africa: a randomized clinical trial. JAMA. 2015;314(17):1808-1817.

41. Detollenaere RJ, den Boon J, Stekelenburg J, et al. Sacrospinous hysteropexy versus vaginal hysterectomy with suspension of the uterosacral ligaments in women with uterine prolapse stage 2 or higher: multicentre randomised non-inferiority trial. BMJ. 2015;351.

42. Stanworth SJ, Estcourt LJ, Powter G, et al. A no-prophylaxis platelettransfusion strategy for hematologic cancers. N Engl J Med. 2013;368(19): 1771-1780.

43. Rahman NM, Pepperell J, Rehal S, et al. Effect of opioids vs nsaids and larger vs smaller chest tube size on pain control and pleurodesis efficacy among patients with malignant pleural effusion: the TIME1 randomized clinical trial. JAMA 2015;314(24):2641-2653.

44. Engert A, Haverkamp H, Kobe C, et al. Reduced-intensity chemotherapy and PET-guided radiotherapy in patients with advanced stage Hodgkin's lymphoma (HD15 trial): a randomised, open-label, phase 3 non-inferiority trial. Lancet. 2012;379(9828):1791-1799.

45. Christiansen EH, Jensen LO, Thayssen P, et al. Biolimus-eluting biodegradable polymer-coated stent versus durable polymer-coated sirolimus-eluting stent in unselected patients receiving percutaneous coronary intervention (SORT OUT V): a randomised non-inferiority trial. Lancet. 2013;381(9867):661-669.

46. Pritchard-Jones K, Bergeron C, de Camargo B, et al. Omission of doxorubicin from the treatment of stage II-III, intermediate-risk Wilms' tumour (SIOP WT 2001): an open-label, non-inferiority, randomised controlled trial. Lancet. 2015;386(9999):1156-1164.

47. Le Henanff A, Giraudeau B, Baron G, Ravaud P. Quality of reporting of noninferiority and equivalence randomized trials. JAMA 2006;295(10):1147-1151.

48. Pocock SJ, Piaggio G, Altman DG, CONSORT Group. Reporting noninferiority trials-reply. JAMA 2013;309(15):1584-1585.

49. O'Dell JR, Mikuls TR, Taylor TH, et al. Therapies for active rheumatoid arthritis after methotrexate failure. N Engl J Med 2013;369(4):307-318.

50. Scott DL, Ibrahim F, Farewell V, et al. Tumour necrosis factor inhibitors versus combination intensive therapy with conventional disease modifying anti-rheumatic drugs in established rheumatoid arthritis: TACIT noninferiority randomised controlled trial. BMJ 2015;350:h1046.

51. Douketis JD, Spyropoulos AC, Kaatz S, et al. Perioperative bridging anticoagulation in patients with atrial fibrillation. $\mathrm{N}$ Engl $\mathrm{J}$ Med 2015;373(9):823-833.

52. Nager CW, Brubaker L, Litman $\mathbf{H J}$, et al. A randomized trial of urodynamic testing before stress-incontinence surgery. N Engl J Med 2012;366(21):1987-1997.

53. Regimbeau J, Fuks D, Pautrat $\mathbf{K}$, et al. Effect of postoperative antibiotic administration on postoperative infection following cholecystectomy for acute calculous cholecystitis: a randomized clinical trial. JAMA 2014;312(2): 145-154.

54. Mulvenna P, Nankivell M, Barton R, et al. Dexamethasone and supportive care with or without whole brain radiotherapy in treating patients with nonsmall cell lung cancer with brain metastases unsuitable for resection or stereotactic radiotherapy (QUARTZ): results from a phase 3, non-inferiority, randomised trial. Lancet 2016;388(10055):2004-2014.

55. Healey JS, Hohnloser SH, Glikson M, et al. Cardioverter defibrillator implantation without induction of ventricular fibrillation: a single-blind, non-inferiority, randomised controlled trial (SIMPLE). Lancet. 2015;385(9970):785-791

56. Reignier J, Mercier E, Le Gouge A, et al. Effect of not monitoring residual gastric volume on risk of ventilator-associated pneumonia in adults receiving mechanical ventilation and early enteral feeding: a randomized controlled trial. JAMA 2013;309(3):249-256.

57. Wangge G, Klungel OH, Roes KC, de Boer A, Hoes AW, Knol MJ. Room for improvement in conducting and reporting non-inferiority randomized controlled trials on drugs: a systematic review. PLoS One 2010;5(10):e13550.

58. Gladstone BP, Vach W. About half of the noninferiority trials tested superior treatments: a trial-register based study. J Clin Epidemiol 2013;66(4):386-396.

59. Djulbegovic B, Kumar A, Glasziou PP, et al. New treatments compared to established treatments in randomized trials. Cochrane Database Syst Rev. 2012;10:Mr000024.

60. Lakens D. Equivalence tests. Soc Psychol Personal Sci. 2017:1948550617697177.

61. Wicherts JM, Veldkamp CLS, Augusteijn HEM, Bakker M, van Aert RCM, van Assen MALM. Degrees of freedom in planning, running, analyzing, and reporting psychological studies: a checklist to avoid $p$ hacking. Front Psychol 2016;7:1832. 